\title{
CARDIAC FAILURE SECONDARY TO INEFFECTIVE BELLOWS ACTION OF THE CHEST CAGE
}

\author{
By JAMES A. FELTMAN, WALTER NEWMAN, ARTHUR SCHWARTZ, \\ DANIEL J. STONE, AND FRANCIS J. LOVELOCK \\ (From the Cardio-Pulmonary Laboratory and the Medical Service at the Bronx Veterans \\ Administration Hospital, Bronx, N. Y.)
}

(Submitted for publication April 7, 1952; accepted June 13, 1952)

Baldwin, Cournand, and Richards (1) have described the following classification of pulmonary insufficiency:

1. Ventilatory

a. Restrictive, viz., pulmonary fibrosis, kyphoscoliosis.

b. Obstructive, viz., pulmonary emphysema.

2. Alveolar-respiratory

a. Distributive, viz., pulmonary emphysema.

b. Diffusional, viz., pulmonary scleroderma.

The two cases to be presented demonstrate ventilatory insufficiency of the restrictive type due in the first instance to primary neuromuscular dysfunction and in the second to chronic pleural disease with resulting calcification and restriction of the underlying parenchyma and diaphragm. Of particular interest was the development of congestive heart failure in both of these patients. These cases represent examples of cardiac failure secondary to an ineffective bellows action of the chest cage without significant intrinsic pulmonary disease.

\section{CASE REPORTS}

\section{Case 1}

This 34 year old white male was well until 1945 when he noted the gradual onset of weakness of the right side of the face. A few months later he began to have bilateral jaw weakness, and difficulty in swallowing food with regurgitation of fluid through the nose. In 1947 there was onset of occasional diplopia. He was admitted to this hospital on three different occasions between January 1947 and November 1948 and on all these admissions showed signs and symptoms of bulbar involvement. These signs and symptoms persisted with little change except for development of dysarthria. In November 1949 he complained of difficulty in maintaining erect posture and he was readmitted for his fourth and final admission. At this time the patient stated he had noted dyspnea on exertion for the preceding one and a half years and during the month before admission, cyanosis of the lips had become apparent.
On admission the patient appeared chronically ill without respiratory distress but with cyanosis of the lips, nailbeds, and skin. There was a marked dysarthria. His chest cage moved very little on normal respiration but he had good expansion with effort and could clear most of his cyanosis with forced breathing. The heart was not remarkable on examination except for a markedly accentuated P2. The lungs were clear to percussion and auscultation. The abdominal examination was within normal limits. Neurological examination revealed the right pupil to be larger than the left, and both reacted sluggishly to light and accommodation. The external ocular movements were normal but he had diplopia in all fields. The disc margins were slightly blurred nasally and he had small hemorrhages about the discs. His gait was normal. There was weakness of the jaw, right facial, palatal, tongue, neck, and shoulder muscles with fasciculations noted in these groups. In addition there was atrophy of the masseters, platysmae, tongue, neck and shoulder muscles. There were a few fasciculations of the triceps muscles but no weakness or atrophy. The deep tendon reflexes of the arms were two to three plus bilaterally and there was greater finger stretch on the left than on the right. The abdominal reflexes were diminished bilaterally. There was no atrophy or weakness of the legs and the sensory examination was normal.

Laboratory examinations including complete blood count, urine analysis, blood chemistries, spinal fluid, serology, chest X-ray, skull plates, and electrocardiographs were all within normal limits.

The patient's neurological status remained unchanged while under observation and adequate nutrition was maintained with tube feeding. Two months after admission a medical consultation was requested because of increasing cyanosis. At this time marked cyanosis was observed without respiratory distress. Expansion of the chest cage during ordinary breathing was minimal but with forced inspiration he was capable of a three inch chest expansion. The lungs were clear and examination of the heart was unremarkable except for an accentuated second pulmonic sound. Abdominal examination was negative. There was no peripheral edema or venous engorgement. The red blood count was 6.75 million with a hemoglobin of 19.4 grams and a hematocrit of $64 \%$. Fluoroscopic examination revealed the trachea to be in the midline and the motion of the bony framework was good. There was normal radiolucency of the lung fields. The range of motion of the right diaphragm was one 
inch; that of the left diaphragm one-half inch. There was no diaphragmatic paralysis. The lung fields were clear. Examination of the heart revealed slight left ventricular rounding, moderate right ventricular enlargement, and marked enlargement of the pulmonary conus. The electrocardiograph demonstrated evidence of right ventricular hypertrophy. Venous pressure was $180 \mathrm{~mm}$. of citrate and decholin circulation time 28 seconds. Because of these findings, the patient was digitalized. There was a five pound diuresis, and the venous pressure and circulation time returned to normal. Following this the patient was studied in the cardiopulmonary laboratory (Table I). Early in June 1950, despite vigorous therapy, he went into intractable congestive heart failure and died.

Autopsy examination revealed the following pertinent findings. There was clubbing of the fingers and moderate subcutaneous edema. The lungs showed a moderate degree of congestion with occasional scattered areas of chronic pneumonitis in the lower lobes, probably on an aspirational basis. There was no gross or microscopic evidence of significant emphysema or fibrosis. The heart was enlarged weighing 500 grams. The coronary arteries, myocardium, endocardium and valvular leaflets were normal. The right ventricular chamber was dilated and the right ventricular wall hypertrophied measuring $1 \mathrm{~cm}$. in thickness. The pulmonic valve ring was dilated measuring $10 \mathrm{~cm}$. in circumference. The right auricle was slightly dilated as was the tricuspid valve ring which measured $14.5 \mathrm{~cm}$. in circumference. The left atrium and mitral valve were normal. The left ventricular wall was slightly hypertrophied to approximately $1.5 \mathrm{~cm}$. in thickness. The aortic valve was normal in size and appearance. The pulmonary arteries showed mild atherosclerotic changes. The gastrointestinal tract, biliary system, genitourinary tract, spleen, pancreas, adrenals, thyroid, lymph nodes, and skeletal system were all normal. There was chronic passive congestion of the liver, spleen and kidneys. The liver weighed 1,750 grams and the spleen 220 grams. The central nervous system, including brain and spinal cord, showed no distinctive lesions on multiple sections with different staining techniques including $\mathrm{Ma}$ hon's. The peripheral nerves were normal.

Despite the negative autopsy findings, it was the opin-

TABLII I

RESULPS Of PULOIURY FUICTIOY STUDIES II CASES 1 AID 2

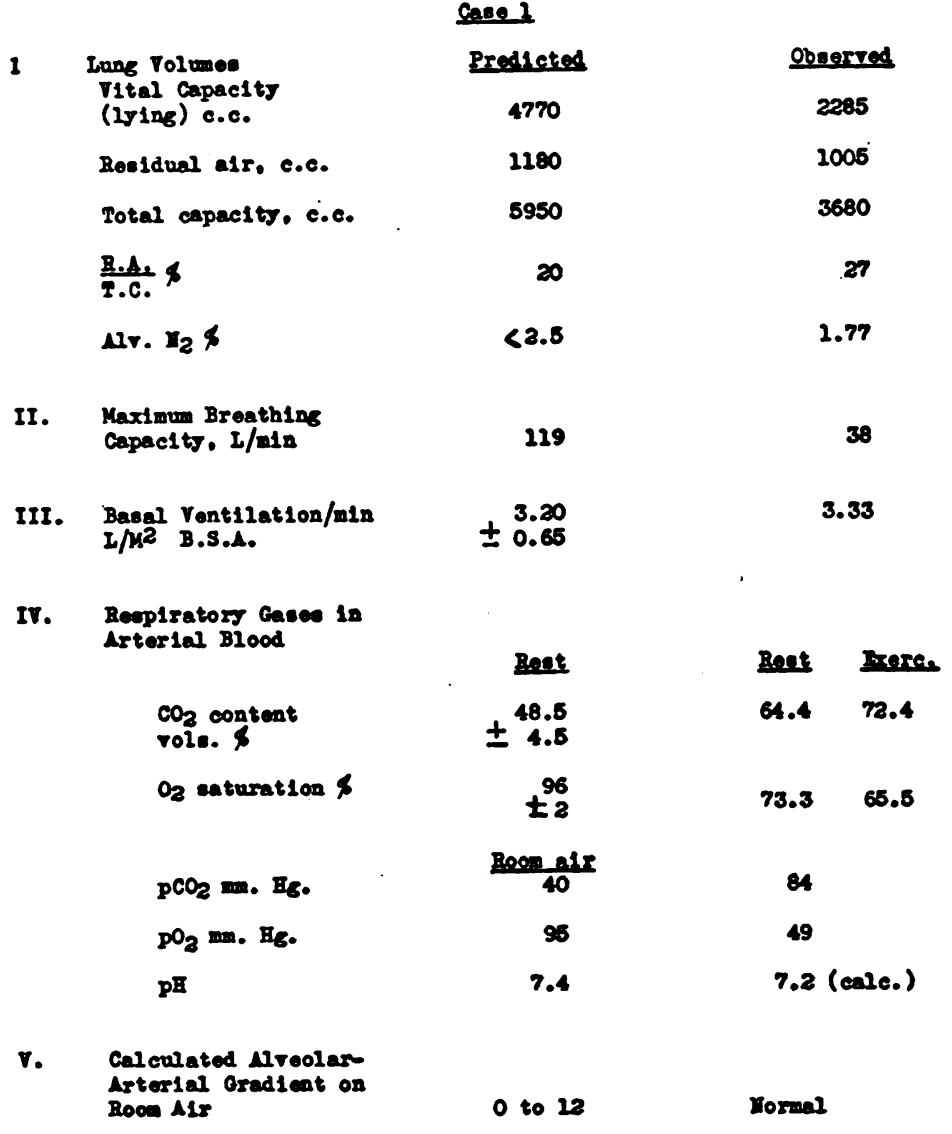


TABrEI (Continued)

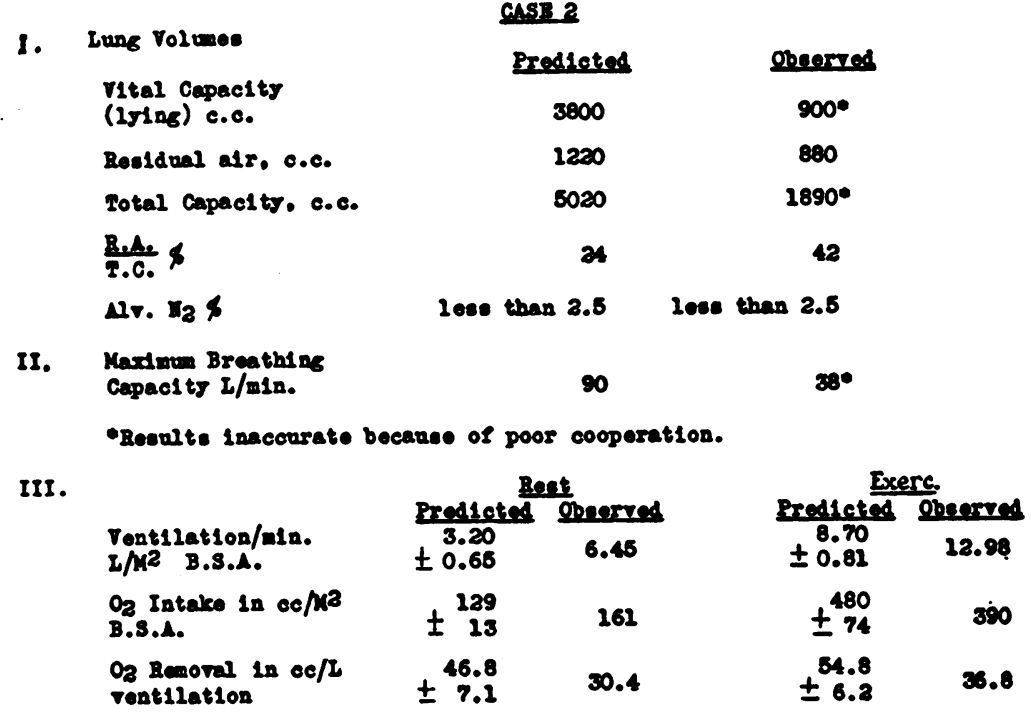

IT. Roeplratory Ganes in

Arterial Blood

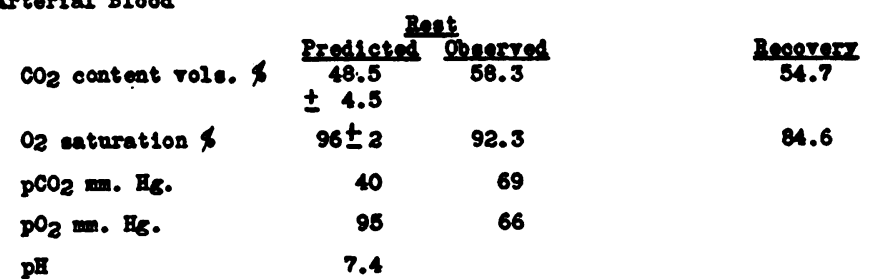

\begin{tabular}{|c|c|}
\hline $\begin{array}{l}\text { Room } \\
\text { alr } \\
\text { breathlne }\end{array}$ & 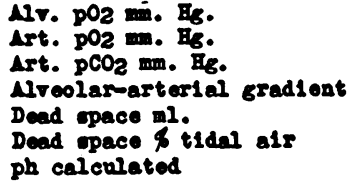 \\
\hline $\begin{array}{l}\text { Bleh } \\
\text { oxsgea } \\
\text { breathing } \\
(29.25 \%)\end{array}$ & 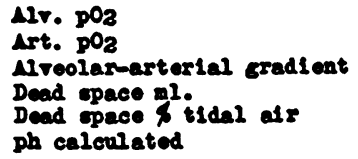 \\
\hline
\end{tabular}

ion of the neurological service that this patient's clinical picture could best be explained by ascribing the changes to amyotrophic lateral sclerosis.

\section{Case 2}

A 56 year old Puerto Rican male was admitted to the hospital because of increased dyspnea and orthopnea for three weeks, and a non-productive cough for a few days. In 1921 and 1925 the patient had "pleurisy." In 1936 he was told that he had "calcifications in his chest." In 1946 he was refused employment because of an abnormal chest X-ray. Three years before admission there was an episode of wheezing and undue shortness of breath which lasted a few days and then ceased spontaneously. During the two years prior to admission there was ankle edema. Because of the increase in dyspnea the patient sought hospitalization.
At the time of admission the patient's temperature was $103^{\circ} \mathrm{F}$., pulse 116 . He was orthopneic and cyanotic. Examination of the chest disclosed poor expansion bilaterally with more limitation on the right. There was dullness over the lower half of the right lung field and at the left base with diminished breath sounds and tactile fremitus over these areas. There were moist rales at the left base with occasional wheezing over the right upper lung field. The second pulmonic sound was accentuated. The liver edge was two finger breadths below the right costal margin, and tender. There was two plus pretibial edema.

The blood count on admission revealed a normal white blood count and differential. The red blood count was 6.0 million, hemoglobin 18.5 grams, and the hematocrit was $62 \%$. An X-ray of the chest disclosed extensive calcification surrounding the lower two-thirds of both lung 

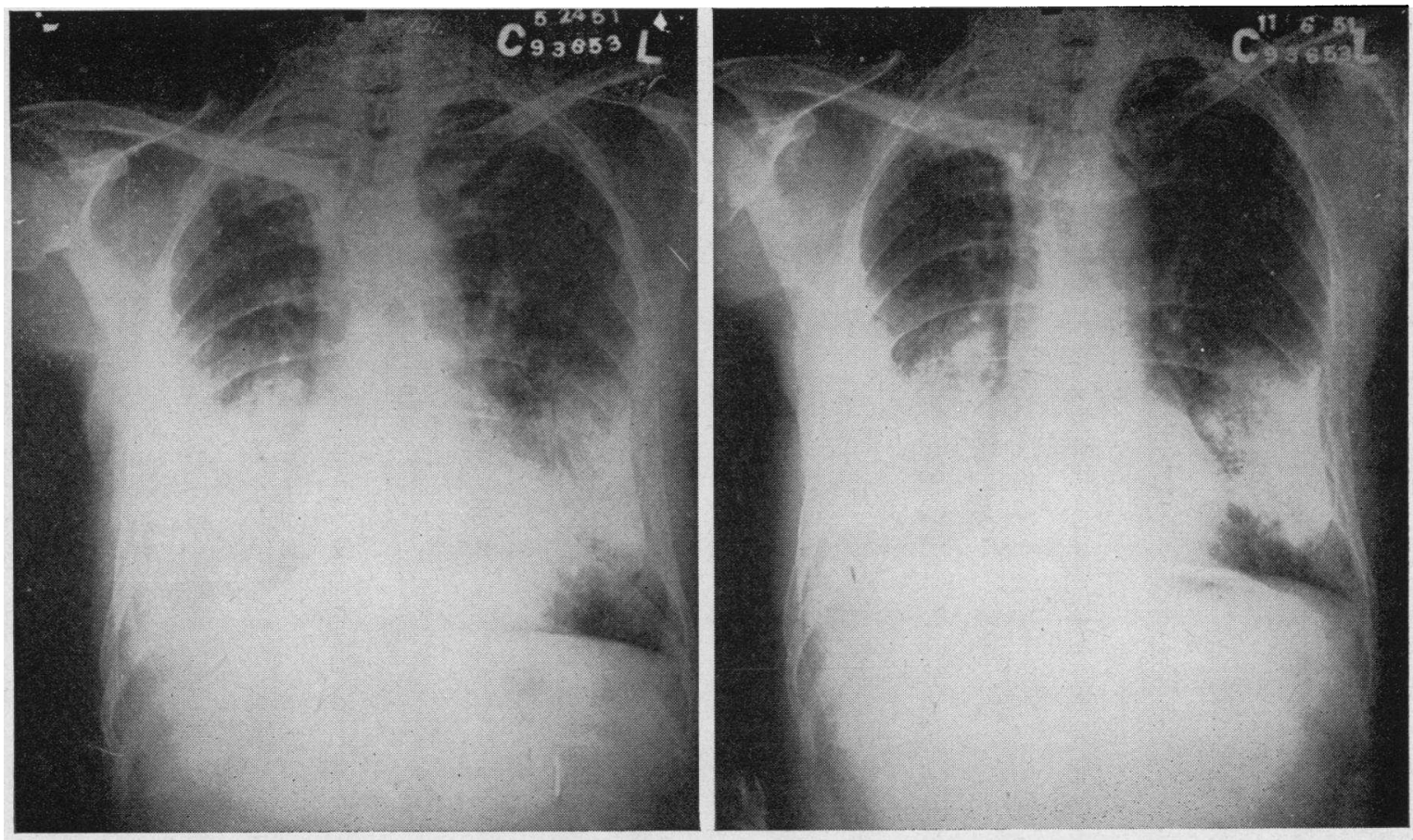

Fig. 1A. Case 2 Demonstrating Calcified Pleuritis and Cardiac Enlargment Before Therapy Fig. 1B. After Therapy Demonstrating Return of Heart Size to Normal

fields and an increase in the transverse diameter of the heart (Figure 1A). An electrocardiogram was consistent with right ventricular hypertrophy. The venous pressure on admission was $280 \mathrm{~mm}$. of citrate.

The patient was treated with penicillin and bed rest for the first five days with no change in the clinical course.
On the fifth hospital day the patient was digitalized, placed on a low salt diet, and oxygen was administered intermittently by mask. Later that day he became semistuporous. He was placed in an oxygen tent and given mercurial diuretics. Shortly thereafter he became comatose although the cyanosis diminished. The stupor per-

TABLE II

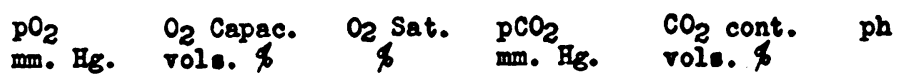

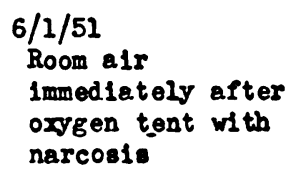

22.2

20.1

19.7

20.2
39.2

96

59.3

62.4
57

68

51
98.9

100 role. $\%$ 
sisted until the patient was removed from the oxygen tent for routine care the following day. Arterial blood studies suggested that carbon dioxide narcosis was responsible for the coma (Table II). In order to combat the $\mathrm{CO}_{2}$ narcosis, artificial respiration with the Pneumatic Balance Resuscitator was initiated. Within a short time the patient was more alert and there was no cyanosis. A phlebotomy was also performed. Artificial respiration was discontinued later that day, and the patient maintained satisfactory clinical progress for the next 48 hours. The blood gas studies reflected this improvement. Although the patient had improved considerably, oxygen therapy was again administered because of the anoxia disclosed by the arterial blood studies. Artificial respiration was reinstituted by means of a Drinker-Collins respirator, and oxygen was administered by nasal catheter. This was continued for one week along with penicillin, bronchodilators and continued digitalis therapy, phlebotomy, low salt diet, and occasional mercurial diuretics. At the end of this period the patient was relatively asymptomatic and the signs of congestive heart failure had disappeared. Follow-up X-rays disclosed that the heart size had returned to normal (Figure 1B). Fluoroscopy revealed the pleural calcifications, no motion of the rib cage, and restriction of diaphragmatic motion. Complete pulmonary function studies were performed one month after recovery from the acute episode (Table I). Three months after discharge the patient was seen in the Follow-up Clinic. He was asymptomatic and capable of climbing two flights of stairs. Digitalis therapy and a low salt diet were continued.

\section{METHODS}

The measurement of residual air volume was done using an open circuit method in which the nitrogen of the lungs is washed out by continuous inhalation of pure oxygen and collected over a period of seven minutes. An index of intrapulmonary mixing was obtained by sampling of the alveolar air at the end of this period (1).

The measurements of lung volumes and maximum breathing capacity were obtained using the spirographic technique (1).

Arterial blood samples were obtained from the brachial artery using an indwelling Cournand-type needle. The blood was collected by the technique described by Riley, Proemmel and Franke (2), using heparin to prevent clotting and a small globule of mercury in the syringe to facilitate mixing. The oxygen content and capacity and the carbon dioxide content were determined on duplicate samples using the Van Slyke-Neill apparatus (3). All samples checked within 0.2 volume $\%$.

The arterial blood tensions of oxygen and of carbon dioxide were determined directly in $\mathrm{mm}$. of mercury, using the direct method of Riley (2). Duplicate measurements were made using two Roughton-Scholander syringes, and all samples checked within $2 \mathrm{~mm}$. of mercury. Expired air samples were collected in duplicate simultaneously with the blood specimens. Oxygen intake and carbon dioxide output were calculated from the percentage of oxygen and carbon dioxide found in these expired air samples, as determined in a Scholander gas analyzer (4). The duplicate measurements checked within $0.04 \%$.

Arterial blood samples and expired air were collected under basal conditions with the patients breathing room air, high, and low oxygen concentrations, each for twenty minute periods when indicated. Finally oxygen saturation was determined after one minute of standard exercise and the expired gas during the exercise was collected in a Douglas bag and analyzed. During the five minute recovery period the expired air was collected and analyzed. The expired air, except during exercise, was collected in the Tissot apparatus and the spirogram attached permitted calculation of the respiratory rate and tidal air under the various conditions. The alveolar-arterial gradients were calculated from the data obtained according to the method of Lilienthal, Riley, Proemmel and Franke (5).

Values for $\mathrm{pH}$ were calculated from line charts of the Henderson-Hasselbalch equation (6).

\section{COMMENTS}

Case 1 disclosed a markedly reduced vital capacity, total capacity and maximum breathing capacity. The residual air and alveolar nitrogen after breathing pure oxygen for seven minutes were normal. The ratio of residual air to total capacity was slightly but not significantly increased and reflected the decrease in total capacity. Thus the lung volume studies revealed principally a reduction in vital capacity which was due primarily to the marked limitation of diaphragmatic excursion. The normal residual air and alveolar nitrogen indicated the absence of any significant degree of emphysema. During the performance of the maximum breathing capacity an element of fatigue was apparent in that the patient was unable to maintain the depth or speed of ventilation uniformly for the 12 second interval. This was probably due to muscle anoxia and weakness associated with chronic disease. In addition, a satisfactory depth of respiration could not be established presumably as a result of impaired diaphragmatic excursion. Finally an adequate speed of ventilation could not be established because of muscular weakness and poor coordination. The total basal ventilation was normal but the effective alveolar ventilation was grossly inadequate as reflected by the high arterial $\mathrm{pCO}_{2}$. Despite the presence of strong stimuli for hyperventilation (high arterial $\mathrm{pCO}_{2}$ and low arterial $\mathrm{pH}$ and $\mathrm{pO}_{2}$ ) the resting ventilation was normal. This would suggest decreased sensitivity 
of the respiratory center to chemical stimuli. In addition, the polycythemia may have been an aggravating factor (7). The inadequate alveolar ventilation resulted from the fact that too large a proportion of the total ventilation was wasted in ventilating the dead space as a result of the shallow breathing. It is of interest to note that this patient's resting ventilation was less than $10 \%$ of his maximum breathing capacity which would account for the absence of dyspnea at rest in the presence of severe anoxemia (1). The arterial $\mathrm{CO}_{2}$ content was elevated but not commensurate with the $\mathrm{pCO}_{2}$ so that the $\mathrm{pH}$ was decreased. The arterial $\mathrm{pO}_{2}$ and oxygen saturation were markedly reduced and the latter decreased even further after standard exercise. These changes were due solely to inadequate alveolar ventilation. The calculated alveolar-arterial gradient on room air, assuming an $R Q$ of 0.8 to 0.9 , was normal. Because of the patient's muscle weakness he was unable to keep a mouthpiece in place for prolonged periods of time, and the $R Q$ of 0.8 to 0.9 was therefore assumed.

Case 2 demonstrated marked decrease in all lung volumes. The observed figures for the vital capacity, total capacity and maximum breathing capacity probably were inaccurate since the patient did not appear to cooperate fully during the performance of these tests. The ratio of residual air to total capacity was high due to the fact that the total capacity reduction was out of proportion to that of the residual air. The maximum breathing capacity was reduced. These changes were primarily due to the chronic constrictive calcific pleuritis which interfered with the bellows action of the chest cage. The high ratio of residual air to total capacity was not a reflection of emphysema since the alveolar nitrogen was less than $2.5 \%$. The tracings showed no graphic evidence of a prolonged expiratory phase. There was no evidence for impairment of gas exchange. Ventilation studies demonstrated that the patient was hyperventilating at rest and during exercise, presumably as a response to the high arterial $\mathrm{pCO}_{2}$ and anoxia. This patient's calcific constrictive pleuritis markedly reduced his effective alveolar ventilation. Thus, his respirations were rapid but shallow and probably did little more than wash out the dead space. This caused a high arterial $\mathrm{pCO}_{2}$ and $\mathrm{CO}_{2}$ content and anoxia. There was no indication of significant abnormalities of gas exchange since the gradient studies were within normal limits $(5,8)$.

The patient was admitted with an acute pulmonary infection. This was enough to accentuate the anoxia and thereby increase the pulmonary hypertension and precipitate cardiac failure. $\mathrm{He}$ was treated with antibiotics, bronchodilators, digitalis, phlebotomy, and oxygen. The use of continuous oxygen therapy in patients with chronic anoxia has been known to produce $\mathrm{CO}_{2}$ narcosis (9-13) and this patient was a vivid example of this phenomenon (Table II). The Pneumatic Balance Resuscitator probably represented a life saving procedure at this time. The use of the respirator permitted continuous use of oxygen therapy without the threat of the return of $\mathrm{CO}_{2}$ narcosis. This will be discussed in another paper (14).

\section{DISCUSSION}

We have presented two patients whose primary difficulty was a defective bellows action of the chest cage. In the first case this was due to disease of the neuromuscular system. In the second case the patient had extensive calcific pleuritis of undetermined etiology which interfered with the mobility of the thoracic wall. In the latter instance there undoubtedly existed some degree of pulmonary

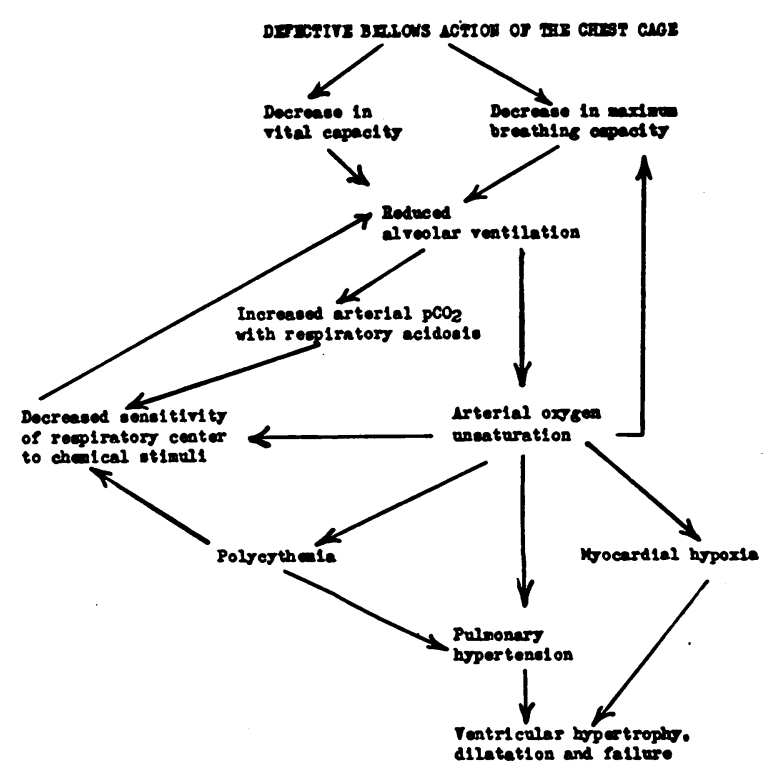

Fig. 2. Sequence of Events Which May Have Occurred in Two Cases with an Ineffective Bellows Action of the Chest Cage 
disease, probably on a tuberculous basis. In view of the entire clinical picture and pulmonary function studies, we are inclined to believe that parenchymal disease contributed little if any to the abnormalities observed.

In both these patients (Figure 2) the defective bellows action resulted in a reduction in vital capacity, maximum breathing capacity, and ineffective alveolar ventilation manifested by a high arterial $\mathrm{pCO}_{2}$ and arterial oxygen unsaturation. The anoxia was probably a stimulus for the production of the polycythemia (15). The hypercapnia, anoxia, and polycythemia all represented factors favoring medullary center damage which these patients demonstrated. There is evidence to indicate that both polycythemia (7) and anoxia $(16,17)$ may lead to pulmonary hypertension and eventually cardiac failure which these patients also manifested. In both these patients the normal changes in circulatory dynamics associated with the mechanics of respiration were undoubtedly altered (18). No objective studies were made to evaluate these factors.

Finally, it is of interest to correlate the situation in primary polycythemia with the sequence of events in these two cases. In the former instance there is polycythemia which we believe may lead to hypoxemia (7). In the latter we have ineffective bellows action of the chest cage leading to hypoxemia and secondary polycythemia. Evidently, once polycythemia and hypoxemia have occurred, the subsequent tendency to develop congestive heart failure probably depends upon similar mechanisms, viz., myocardial hypoxia and pulmonary hypertension.

\section{SUMMARY}

Two cases of ventilatory insufficiency of the restrictive type resulting in congestive heart failure have been presented. There was no evidence of significant intrinsic pulmonary disease. The sequence of events leading to heart failure are discussed.

\section{ACKNOWLEDGMENTS}

The authors wish to acknowledge their appreciation to Dr. Richard L. Riley for his many helpful comments in the preparation of this manuscript, and to Mrs. Blanche Devlin for her invaluable aid in the laboratory procedures.

\section{REFERENCES}

1. Baldwin, E. de F., Cournand, A., and Richards, D. W., Jr., Pulmonary insufficiency. I. Physiological classification, clinical methods of analysis, standard values in normal subjects. Medicine, 1948, 27, 243.

2. Riley, R. L., Proemmel, D. D., and Franke, R. E., A direct method for determination of oxygen and carbon dioxide tensions in blood. J. Biol. Chem., 1945, $161,621$.

3. Van Slyke, D. D., and Neill, J. M., The determination of gases in blood and other solutions by vacuum extraction and manometric measurements. I. J. Biol. Chem., 1924, 61, 523.

4. Scholander, P. F., Analyzer for accurate estimation of respiratory gases in one-half cubic centimeter samples. J. Biol. Chem., 1947, 167, 235.

5. Lilienthal, J. L., Jr., Riley, R. L., Proemmel, D. D., and Franke, R. E., An experimental analysis in man of the oxygen pressure gradient from alveolar air to arterial blood during rest and exercise at sea level and altitude. Am. J. Physiol., 1946, 147, 199.

6. Van Slyke, D. D., and Sendroy, J., Jr., Studies of gas and electrolyte equilibria in blood. XV. Line charts for graphic calculations by the HendersonHasselbalch equation, and for calculating plasma carbon dioxide content from whole blood content. J. Biol. Chem., 1928, 79, 781.

7. Newman, W., Feltman, J. A., and Devlin, B., Pulmonary function studies in polycythemia vera. Am. J. Med., 1951, 11, 706.

8. Rahn, H., Effects of unequal blood flow and ventilation upon the alveolar-arterial gradient. Federation Proc., 1949, 8, 129.

9. Barach, A. L., and Richards, D. W., Jr., Effects of treatment with oxygen in cardiac failure. Arch. Int. Med., 1931, 48, 325.

10. Barach, A. L., Effect of low and high oxygen tensions on mental functioning. J. Aviation Med., 1941, 12, 30.

11. Comroe, J. H., Jr., Bahnson, E. R., and Coates, E. O., Jr., Mental changes occurring in chronically anoxemic patients during oxygen therapy. J. A. M. A., 1950, 143, 1044.

12. Davies, C. E., and Mackinnon, J., Neurological effects of oxygen in chronic cor pulmonale. Lancet, 1949, 2, 883.

13. Donald, K., Neurological effects of oxygen. Lancet, 1949, 2, 1056.

14. Stone, D. J., Schwartz, A., Newman, W., Feltman, J. A., and Lovelock, F. J., The precipitation by pulmonary infection of anoxia, cardiac failure, and respiratory acidosis: pathogenesis and treatment. Am. J. Med., to be published. 
15. Viault, Sur la quantité d'oxygène contenue dans le sang des animaux des hauts plateaux de l'Amérique du Sud. Compt. rend. Acad. Sc., 1891, 112, 295.

16. Motley, H. L., Cournand, A., Werko, L., Himmelstein, A., and Dresdale, D., The influence of short periods of induced acute anoxia upon pulmonary artery pressures in man. Am. J. Physiol., 1947, $150,315$.
17. Westcott, R. N., Fowler, N. O., Scott, R. C., Hauenstein, V. D., and McGuire, J., Anoxia and human pulmonary vascular resistance. J. Clin. Invest., 1951, 30, 957.

18. Lawson, H. D., Bloomfield, R. A., and Cournand, A., The influence of the respiration on the circulation in man. Am. J. Med., 1946, 1, 315. 